\title{
INTERGRANULE FUSION IN RAT PARS INTERMEDIA CELLS
}

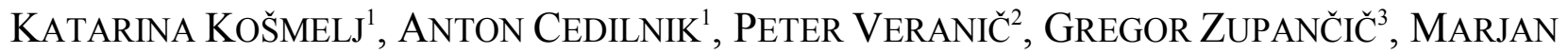 \\ RUPNIK $^{3}$, LAURA KOCMUR-BOBANOVIĆ ${ }^{3}$ AND ROBERT ZOREC ${ }^{3}$
}

${ }^{1}$ Biotechnical Faculty, University of Ljubljana, Jamnikarjeva 101, 1111 Ljubljana, Slovenia, ${ }^{2}$ Institute of Cell Biology, Medical School, Lipičeva 5, 1001 Ljubljana, Slovenia, ${ }^{3}$ Laboratory of Neuroendocrinology-Molecular Cell Physiology, Institute of Pathophysiology, Medical School, P.O. Box 2211, 1001 Ljubljana, Slovenia e-mail: robert.zorec@pafi.mf.uni-lj.si

(Accepted April 26, 2001)

\begin{abstract}
Using electron microscopy, we studied the morphology of secretory granules in rat pars intermedia cells. We found figures of apparent intergranule fusion, characterized by a tight association of two granules. The fusion was detected in around $2 \%$ of all measured granules, indicating a low occurrence of intergranule fusion. To study whether intergranule fusion affects the distribution of granule diameters a simple probabilistic model was developed. It is based on the theory that larger granules are formed by fusion of two or more spherical granules of fixed size, and that the surface of a newly formed granule is equal to the sum of fused granule membranes. The model accounts for the bias on granule diameter measurements due to sectioning of granules. Although the electron microscopy data strongly indicates the existence of intergranule fusion in rat melanotrophs, this process as modelled in the present work does not contribute to the granule diameter distribution significantly. It is likely that in addition to the fusion of larger granules, other processes, such as fusion of microvesicles, may affect the distribution of granule diameters.
\end{abstract}

Keywords: intergranule fusion, melanotrophs, probabilistic model, rat, secretory granules, stereology.

\section{INTRODUCTION}

Cells from the rat pars intermedia secrete a number of peptides deriving from post-translational processing of pro-opiomelanocortin (POMC), including $\beta$ endorphin, $\alpha$-melanocyte stimulating hormone $(\alpha$ MSH) and adrenocorticotrophin (ACTH, Mains and Eipper, 1979). The formation of peptide-containing secretory granules begins with the condensation of secretory products within the lumen of the trans-Golgi network, followed by budding of immature secretory granules (Tooze, 1991). During the maturation process the size of secretory granules is increasing (Farquhar et al., 1978; Tooze et al., 1991), and in rat melanotrophs their cargo is undergoing metabolic changes (Tanaka et al., 1991). Larger granules possibly result from fusion between smaller granules of unitary size (Alvarez de Toledo and Fernandez, 1990; Hartmann et al., 1995). The size of larger granules may also be influenced by the fusion of microvesicles with secretory granules. Microvesicles are thought to be trafficking between secretory granules and other compartments (Tooze and Tooze, 1986; Komuro et al., 1987).
The size of the secretory granules in rat pars intermedia depends on physiological state of the animal. It is reduced when the animal is pretreated with bromocriptine (Bäck, 1989). However, an increase of granules was reported in hypersecretory melanotrophs. It could be due to mechanisms operating at an early stage of granule formation (Bäck and Soinila, 1994).

In the present study we use electron microscopy to examine the morphology of secretory granules in rat pars intermedia cells. We detected some apparent intergranule fusions. The main objective of our study was to find out if granule growth is associated with fusion of unitary granules (Alvarez de Toledo and Fernandez, 1990; Hartmann et al., 1995). If this is the dominant process in granule diameter growth in rat melanotrophs, robust peaks in the multimodal distribution of granule diameters are anticipated.

The reported distributions of granule profile diameters in rat melanotrophs are heterogeneous (Bäck, 1989; Zupančič et al., 1994). The previous electrophysiological experiments (Zupančič et al., 1994) were not sufficiently sensitive, therefore we 
studied secretory granule morphology by electron microscopy. To analyse the granule diameter distribution we developed a simple probabilistic model assuming that larger granules are formed by fusion of two or more spherical granules of fixed size, and that the surface of a newly formed granule is equal to the sum of fused granule membranes. The model also accounts for the bias due to the sectioning of embedded specimens prepared for electron microscopy, a standard stereological problem.

\section{MATERIAL AND METHODS}

\section{CELL PREPARATION, ELECTRON MICROSCOPY AND MEASUREMENTS OF GRANULE DIAMETERS}

After ether anaesthesia animals (male Wistar rats, 200 to $300 \mathrm{~g}$ ) were killed by decapitation. Pars intermedia of each animal was carefully dissected from the pituitary gland and divided into 4 to 6 pieces of tissue in Earl's Balanced Salt Solution (EBBS, Sigma Chemical Co., USA). Tissue pieces were maintained in a culture medium (Rupnik and Zorec, 1992), placed in an incubator $\left(36^{\circ} \mathrm{C}, 4.6 \% \mathrm{CO}_{2}\right)$ for 24 hours, and then fixed in buffered $(\mathrm{pH} \mathrm{7.3)} 0.5 \%$ glutaraldehyde with $0.5 \%$ tannic acid for 30 minutes at room temperature. After that the tissue was fixed for an hour in buffered (pH 7.3) 2.5\% glutaraldehyde without tannic acid. After the postfixation in $2 \%$ osmium tetroxide with $3 \%$ ferrocyanide tissue pieces were immersed in $1 \%$ water solution of uranil acetate for 30 minutes in darkness. After dehydration the tissue was embedded in Epon 812 (Medium grade, London Resin Co, U.K.). Ultrathin sections $(70 \mathrm{~nm})$ were cut with a LKB ultramicrotom and examined with a Jeol T8 transmission electron microscope.

Diameters of secretory granule profiles were measured in $\mathrm{mm}$ from electron micrographs, enlarged to the scale of 126000 . The smallest detectable profile diameter was $8 \mathrm{~mm}(64 \mathrm{~nm})$. The accuracy of the data readings was $\pm 1 \mathrm{~mm}(8 \mathrm{~nm})$. Some of the profiles appeared non-spherical, their maximal diameter was measured.

\section{THEORETICAL MODEL \\ Sectioning Process}

Let us assume a population of sphere granules all having an equal diameter $D$. Granules are cut by parallel random planes (Fig. 1A). For each granule, parallel cuts are uniformly distributed on the granule diameter. Diameters of the obtained sphere profiles (d) are measured.

A

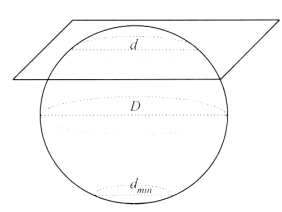

B
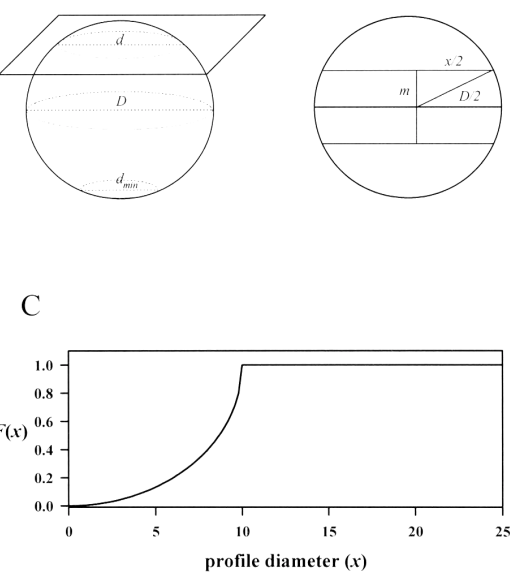

D

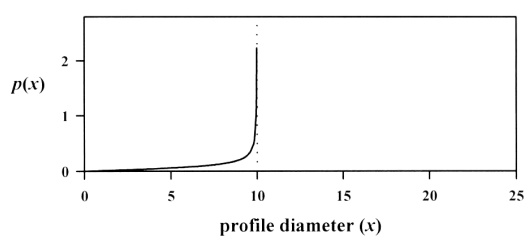

Fig. 1. A presents a diagram of sectioning a spherical secretory granule with diameter $D$. On each sphere profile its diameter $d$ is measured. The smallest detectable profile diameter is $d_{\text {min. }}$. $\boldsymbol{B}$ The distribution function $F(x)$ is obtained on the basis of this plot, from the right-angled triangle with sides $D / 2, m$ and x/2 (see Material and Methods, Theoretical Models, Sectioning Process). C A distribution functioon $F(x)$ for profile diameters is $D=10$ (arbitrary units). The smallest detectable profile diameter is $d_{\min }=0$. D Probability density function $p(x)$ for profile diameters. The granule diameter is $D=10$ (arbitrary units). The smallest detectable profile diameter is $d_{\min }=0$. 
Profile diameter $d$ can be regarded as a random variable with values ranging from 0 to $D$. Its distribution function ${ }^{1}, F(x), 0<x<D$ is obtained geometrically from a right-angled triangle with sides $D / 2, m$ and $x / 2$ (Fig. 1B). It is:

$$
F(x)=1-\frac{2 m}{D}=1-\frac{\sqrt{D^{2}-x^{2}}}{D} .
$$

Profile diameters smaller than a particular value can not be detected reliably in experimental work. Let us denote the smallest detectable diameter $d_{\min }$; its value is known from the experimental technique. Then the distribution function is:

$$
F(x)=1-\sqrt{\frac{D^{2}-x^{2}}{D^{2}-d_{\min }^{2}}}, d_{\min }<x<D .
$$

Differentiation of Eq. 1 gives the probability density function ${ }^{2} p(x)$ :

$$
p(x)=\frac{x}{\sqrt{\left(D^{2}-d_{\min }^{2}\right)\left(D^{2}-x^{2}\right)}}, d_{\min }<x<D
$$

A pole at $x=D$ should be noted. For illustration we present $F(x)$ and $p(x)$ on Fig. 2. It turns out that the expected value of $d$ is:

$$
\mathrm{E}(d)=\int_{d_{\min }}^{D} x p(x) d x=\frac{d_{\min }}{2}+\frac{D^{2}}{2 \sqrt{D^{2}-d_{\min }^{2}}} \arccos \frac{d_{\min }}{D}
$$

Since $\mathrm{E}(d)$ is an increasing function of $d_{\min }$, its infimum is:

$$
\inf \mathrm{E}(d)>\frac{\pi}{4} D
$$

Thus, the average value of profile diameters is approximately $\pi / 4$ of $D$ for small $d_{\min }$, consistent with Weibel (1979). These results provide theoretical background for the graphical methods commonly used in stereology.

\section{Fusion Effect Model}

This model describes the fusion theory as follows. The smallest granules, i.e., unit granules, fuse into double, triple, ... granules. The maximal granule size type $K$ is unknown. For simplicity reasons we assume that the unit granules are spherical, their diameter $D_{1}$ fixed but unknown. Following the intergranule fusion theory, the surface of the granule size type $k$, say $S_{k}$,

\footnotetext{
${ }^{1}$ Distribution function $F(x)$ gives the probability that a random variable $X$ takes a value less than $x, F(x)=P(X<x)$.

${ }^{2}$ Probability density function $p(x)$ is obtained as the derivative of $F(x)$.
}

is, $S_{k}=k S_{1}, k=1, \ldots, K$ and consequently its diameter $D_{k}=\sqrt{k} D_{1}$.

Fusion is a rare event. Therefore, we assume that the probability of fusion into a granule type $k, p_{\mathrm{k}}$, follows a finite Poisson-like distribution:

$$
p_{k}=\alpha_{K} \frac{\lambda^{k-1}}{(k-1) !}, \quad k=1,2, \ldots K,
$$

Probability $p_{k}$ describes the fraction of type $k$ granules. It can be shown that $\lambda$ is the unknown parameter representing the expected granular size type in the following way:

$$
\mathrm{E}_{K}(k) \approx 1+\lambda,
$$

and $\alpha_{K}$ is the normalizing constant:

$$
\alpha_{K}=\left(1+\frac{\lambda}{1}+\frac{\lambda^{2}}{2}+\ldots+\frac{\lambda^{K-1}}{(K-1) !}\right)^{-1} .
$$

These granules undergo the sectioning process described previously. i.e. granules are cut by parallel random planes. For each granule, parallel cuts are uniformly distributed on the granule diameter. Diameters of the obtained sphere profiles are measured. We denote by $q_{k}$ the probability that a granule of type $k$ is hit by a random plane. This probability is proportional to the granule's diameter:

$$
q_{k} \propto \sqrt{k} D_{1}
$$

and to the proportion of granules of type $k$ :

$$
q_{k} \propto p_{k}
$$

It turns out, that $q_{k}$ is as follows:

$$
q_{k}=\frac{p_{k} \sqrt{k}}{\sum_{k=1}^{K} p_{k} \sqrt{k}}, \quad k=1, \ldots, K
$$

Following Eq. 1, the distribution function for any granular size type $k$ is:

$F_{k}(x)=1-\sqrt{\frac{k D_{1}^{2}-x^{2}}{k D_{1}^{2}-d_{\text {min }}^{2}}}, \quad d_{\min }<x<\sqrt{k} D_{1}$.

Pooling together all the granular size types with the corresponding probabilities $q_{k}$ (Eq. $4 \mathrm{~b}$ ) gives the global distribution function $G(x)$ : 


$$
G(x)=\sum_{k=1}^{K} q_{k} F_{k}(x) .
$$

$G(x)$ is of saw-tooth shape. Fig. 2 illustrates $G(x)$ graphically. Relative cumulative function estimates the distribution function $G(x)$, which is nonlinear.

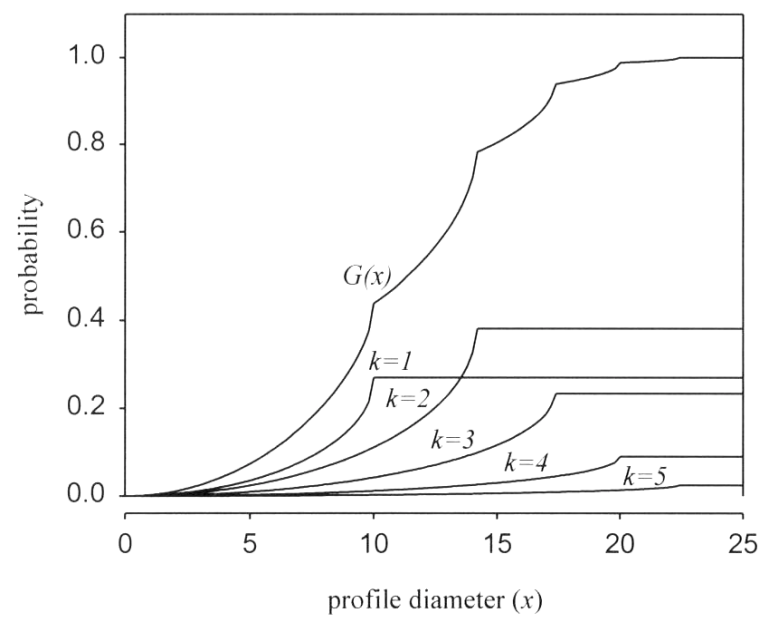

Fig. 2. Presents distribution function $G$ and its components based on Model 1. The following values of parameters are taken into account: $D_{I}=10$ (arbitrary units), $\lambda=1, K=5$. The smallest detectable profile diameter is $d_{\min }=0$.

The parameters to be estimated are $D_{1}$ and $\lambda$. Their starting values can be estimated from the empirical distribution of profile diameters: starting value for $D_{1}$ corresponds to the abscissa of the first observable peak, $\lambda$ to the granular size type with the highest frequency. A number of numerical techniques may be applied to find the least squares estimates commonly used in regression models (Everitt, 1987). A simpler procedure is a grid-search technique. For each couple $\left(D_{10}, \lambda_{0}\right)$ in a fine two-dimensional grid (its borders are suggested by the data) we calculate the residual sum of squares RSS. Couple $\left(D_{10}, \lambda_{0}\right)$ with the smallest RSS is considered to be 'the best'. The fitting procedure for $D_{1}$ and $\lambda$ is repeated for each value of $K$ in the reasonable range. The corresponding values of RSS are compared.

\section{Intergranule Fusion}

The pars intermedia of rat pituitary presents a homogeneous tissue formed by closely associated polygonal cells with ovoid nuclei. Secretory granules of various electron densities fill most of the cytoplasm, and are stained positively with an anti $\beta$ endorphin antibody (not shown). Apparent intergranule fusions were observed (Fig. 3, see arrow) in the pars intermedia preparations taken from 7 animals. The larger granule was typically electron lucent, the other being electron dense. It was usually localized to one part of the larger granule, or sometimes as a spherical structure inside the larger granule (Fig. 3, see arrowhead). The frequency of such events was relatively low. Out of 3144 secretory granules measured in 24 cells only 80 intergranule fusions were detected.

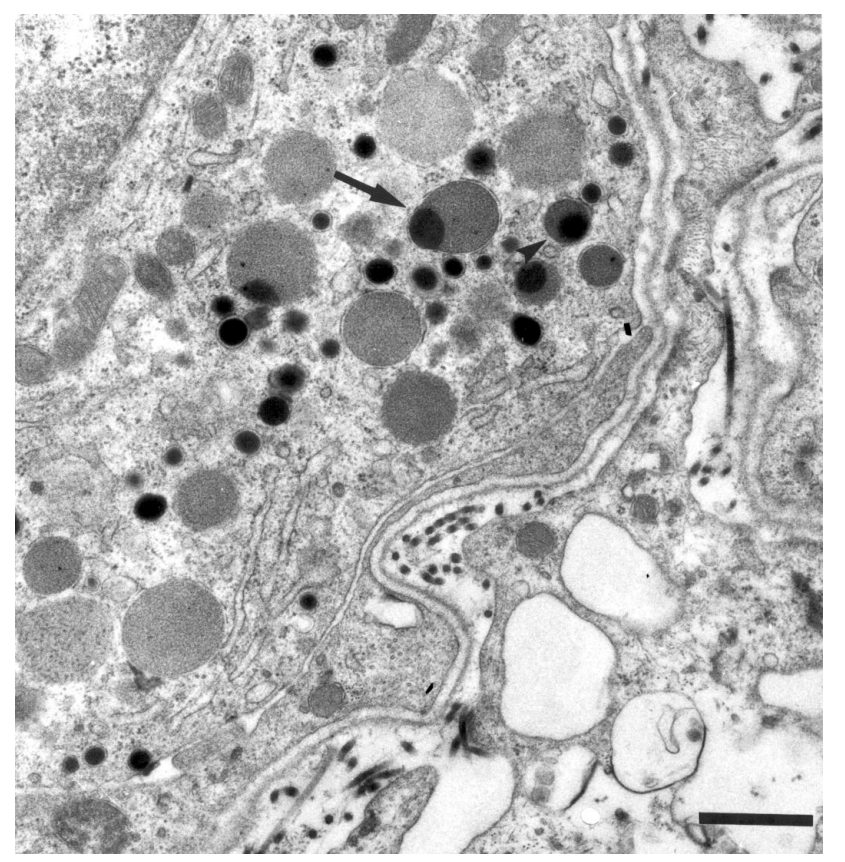

Fig. 3. Representative electron micrograph of a rat pars intermedia cell. Arrow indicates a tight association between two granules at an early step of (or just after) intergranule fusion. Arrowhead points to an electron dense inclusion in a secretory granule, possibly formed after a completion of intergranule fusion. Bar denotes $1000 \mathrm{~nm}$.

\section{Distribution of Granule Diameters}

Fig. 4A presents an empirical distribution of secretory granule profile diameters measured from 11 cells of the same animal. The distribution is skewed to the right consistent with the previous report (Zupančič et al., 1994). Six other preparations show a similar distribution. The comparison of distributions from a single cell (Fig. 4B) and pooled cells of the same animal (Fig. 4A) indicates that the two distributions are similar. A potential multimodality may be anticipated in Fig. 4A. 
A

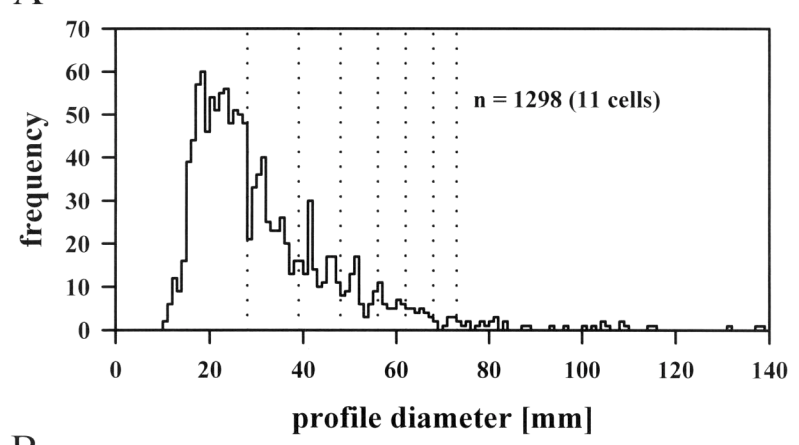

B
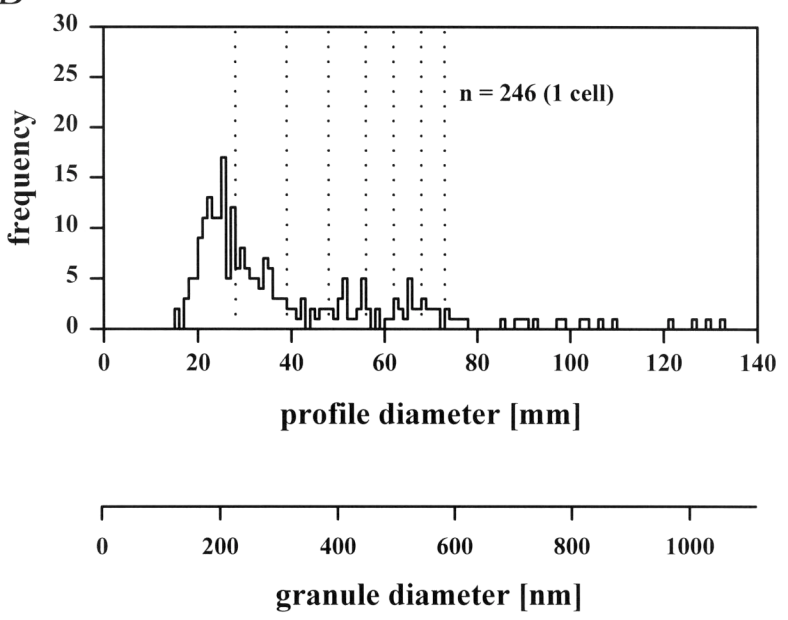

Fig. 4. Frequency distribution of secretory granule profiles obtained from 11 cells (Fig. 4A) and from 1 cell (Fig. 4B), all taken from the same animal. Dashed vertical lines represent values of estimated secretory granule diameters (see Table 2).

\section{Model Fitting}

We tested whether the model presented in the Material and Methods could be a reasonable description of the empirical distribution presented on Fig. 5A. The measured profile diameters range from 11 to $139 \mathrm{~mm}$ (87 to $1103 \mathrm{~nm}$ ). In the final statistical analysis seventeen granules $(1.3 \%)$ with profile diameter larger than $84(667 \mathrm{~nm})$ were not included. The mean profile diameter for the remaining 1281 granules is $31.3 \mathrm{~mm}(248 \mathrm{~nm})$, the standard deviation $13.9 \mathrm{~mm}$ $(110 \mathrm{~nm})$. The abscissa of the first possible peak is somewhere within 19-28 mm (151-222 nm).

\section{Fusion Effect Model}

As described previously we used a simple gridsearch procedure to find the estimates for $D_{1}$ and $\lambda$. The grid was as follows: $10 \mathrm{~mm}$ to $30 \mathrm{~mm}$ with step $0.25 \mathrm{~mm}$ for $D_{1}$, and 0.10 to 4 with step 0.05 for $\lambda$. The search was repeated for each $K=2,3, . .9$. The estimates accepted are: $D_{1}=27.75 \mathrm{~mm}, \lambda=0.65$. The RSS does not change considerably after $K=4$ (see Table 1).

Table 1. Residual sum of squares (RSS), estimate for granule diameter $D_{1}$ (in $\mathrm{mm}$ ) and $\lambda$ for the maximal granular size type $K=2,3, \ldots, 7$.

\begin{tabular}{c|ccc}
\hline$K$ & RSS & $D_{1}(\mathrm{~mm})$ & $\lambda$ \\
\hline 2 & 0.3919 & 30.00 & 0.65 \\
3 & 0.2648 & 28.50 & 0.65 \\
4 & 0.2226 & 27.75 & 0.65 \\
5 & 0.2120 & 27.75 & 0.65 \\
6 & 0.2101 & 27.75 & 0.65 \\
7 & 0.2099 & 27.75 & 0.65 \\
\hline
\end{tabular}

According to the results obtained the probability of appearance of unit granules is 0.522 , the probability for double granules is 0.339 , the probability of triple granules 0.110 . The fraction of other granule types is negligible (see Table 2).

Table 2. Granule diameters $D_{k}$ and its probability $p_{k}$ for each granule size type $k=1,2, \ldots 7$. The results are based on the estimates: $D_{1}=27.75 \mathrm{~mm}, \lambda=, K=7$ (see Table 1).

\begin{tabular}{l|ccccccc}
\hline$k$ & 1 & 2 & 3 & 4 & 5 & 6 & 7 \\
\hline$D_{\mathrm{k}}(\mathrm{mm})$ & 28 & 39 & 48 & 56 & 62 & 68 & 73 \\
$p_{\mathrm{k}}$ & 0.5220 & 0.3393 & 0.1103 & 0.0239 & 0.0039 & 0.0005 & 0.0001 \\
\hline
\end{tabular}

The comparison of the relative cumulative function with its fit $G$ (Fig. 5) shows that the model describes the empirical distribution considerably well up to approximately $37 \mathrm{~mm}$, deviations increase afterwards. Fig. 4 presents dashed vertical lines, representing the expected position of granule diameters. Agreement of the expected positions and the actual peaks is not satisfactory. 


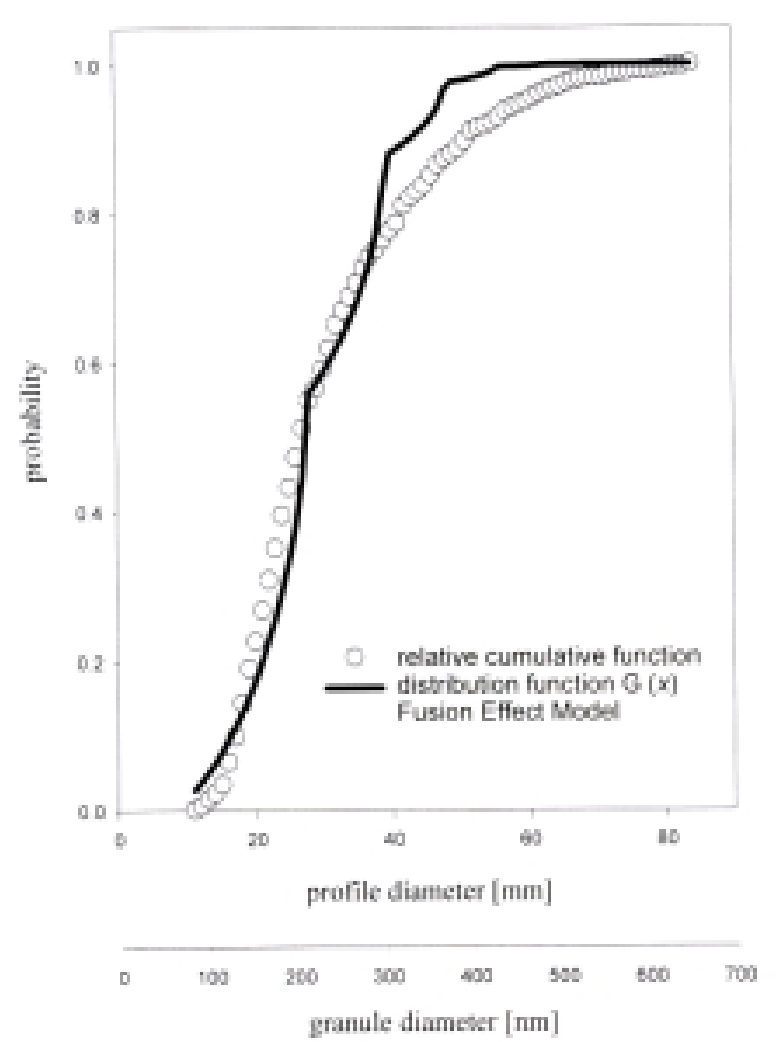

Fig. 5. Comparison of empirical data with the model in which intergranule fusion plays a role in granule size determinantion. Relative cumulative frequency (open circles) and its fit $G$ (line) obtained on the basis of the following estimates: $K=7, D_{1}=27.75$ $\mathrm{mm}, \lambda=0.65$. Residual sum of squares is 0.2099.

\section{DISCUSSION}

Using electron microscopy, we studied the secretory granule morphology of rat pars intermedia cells. Morphological features representing intergranule fusion (Fig. 3) are among the clearest examples in comparison to those found in literature. The initiation of this process is indicated by a close association of two granules: a large, electron lucent, and a smaller, electron dense (Fig. 3, see arrow). On the other hand, an electron dense granule localized inside an electron lucent granule was also observed, indicating a completion of intergranule fusion (Fig. 3, see arrowhead).

The results are consistent with the previous studies. Farquhar et al. (1978) showed that packaging of prolactin into secretory granules involves aggregation of immature (smaller) granules forming larger ones. Fumagalli and Zanini (1985) describe that some mature secretory granules, located in somatomammotrophs, contain growth hormone and prolactin. Both are segregated in different portions of the granule, they possibly originate from intergranule fusion. Moreover, the maturation of secretory granules in $\mathrm{PC} 12$ cells is associated by an increase in diameter from an average of about $80 \mathrm{~nm}$ to about $120 \mathrm{~nm}$ possibly due to intergranule fusion (Tooze et al., 1991).

Morphological features of intergranule fusion in pars intermedia cells (Fig. 3) may also be involved in the maturation of secretory granules. Different electron densities of the fused granules could be attributed to the metabolic status of their cargo changing during the process of granule maturation. Electron dense granules probably represent immature granules. Tanaka et al. (1991) showed immunocytochemically that POMC was selectively found in electron dense granules, whereas $\alpha$ MSH in electron lucent granules. This result is consistent with the view that these granules are biosynthetic and storage organelles (Mains et al., 1987).

In electrophysiological studies intergranule fusion was interpreted to contribute to the multimodal distribution of amplitudes of unitary exocytic membrane capacitance events, which report the size of fusing granules (Alvarez de Toledo and Fernandez, 1990; Hartmann et al., 1995). To test whether this mechanism also affects the distribution of granule size in rat melanotrophs, we developed a simple probabilistic model predicting the formation of larger granules is due to the fusion of unitary granules. The model accounts for the sectioning of secretory granules (Weibel, 1979). This problem was solved analytically. The peaks in the distribution of measured profiles are expected to occur in the sequence $D, D \sqrt{2,} D \sqrt{3, \ldots}$ (see Theoretical Model, see Fig. 4B, dashed vertical lines), however the empirical distribution does not confirm that satisfactory.

It should be noted that in addition to tissue sectioning, examination by electron microscopy incorporates other features introducing a nonestimable bias in the data. For example: sections are not infinitely thin, granule profiles are not always sharp, nonspherical granules were also observed in our study. The assumption that unitary granules are of a fixed size is an oversimplification. Other processes, such as fusion of microvesicles with secretory granules (Tooze and Tooze, 1986; Komuro et al., 1987), may additionally affect the distribution of secretory granule diameters.

To sum up, our electron micrographs reveal compelling evidence in support of intergranule fusion in rat pars intermedia cells. In an attempt to analyze the contribution of intergranule fusion to secretory granule growth, we consider the hypothesis that larger granules result from fusion between smaller granules 
of unitary size (Alvarez de Toledo and Fernandez, 1990; Hartmann et al., 1995). We developed a simple probabilistic model to explain this type of fusion and electron microscopy data acquisition, simultaneously. The data obtained do not give evident results, the fit is unsatisfactory. Therefore we conclude that further work has to be undertaken to get to a more conclusive answer about the fusion theory.

\section{ACKNOWLEDGEMENTS}

This work was supported by the grant \#J3-6207381 from The Ministry of Science and Technology, The Republic of Slovenia. We thank S. Grilc for cell cultures and N. Kuzmin for improving our English.

\section{REFERENCES}

Alvarez de Toledo G, Fernandez JM (1990) Patch-Clamp Measurements Reveal Multimodal Distribution of Granule Sizes in Rat Mast Cells. J Cell Biol 110: 1033-9.

Bäck N (1989) The Effect of Bromocriptine on the Intermediate Lobe of the Rat Pituitary: an Electron Microscopic, Morphometric Study. Cell Tissue Res 255:405-10.

Bäck N, Soinila S, Virtanen I (1993) Endocytotic Pathway in the Melanotroph of the Rat Pituitary. Histochem J 25:133-9.

Bäck N, Soinila S (1994) Regulation of Secretory Granule Formation in Chronically Hypersecretory Melanotrophs in the Rat Pituitary. Cell Tissue Res 275:339-44.

Everitt BS (1987) Introduction to Optimization Methods and Their Application in Statistics. Chapman and Hall, 42-8.

Farquhar MG, Reid J, Daniell LW (1978) Intracellular Transport and Packaging of Prolactin: A Quantitative Electron Microscope Autoradiographic Study of Mammotrophs Dissociated from Rat Pituitaries. Endocrinology 102:296-311.

Fumagalli G, Zanini A (1985) In Cow Anterior Pituitary, Growth Hormone and Prolactin Can Be Packaged in Separate Granule of the Same Cell. Cell Biol 100:201924.
Hartmann J, Scepek S, Lindau M (1995) Regulation of Granule Size in Human and Horse Eosinophils by Number of Fusion Events among Unit Granules. J Physiol (Lond) 484.1:201-9.

Komuro M, Kiuchi Y, Shioda T (1987) Membrane Modification During Secretory Granule Formation in Rat Somatotrophs. Eur J Cell Biol 43:98-103.

Mains RE, Eipper BA (1979) Synthesis and Secretion of Corticotropins, Melanotropins and Endorphins by Rat Intermediate Pituitary Cells. J Biol Chem 254:7885-94.

Mains RE, Cullen EI, May V, Eipper BA (1987) The Role of Secretory Granules in Peptide Biosynthesis. Ann NY Acad Sci 493:278-91.

Rusakov DA (1993) Estimation of the Size of Closed Cell Elements from Analysis of Their Random Plane Sections. Biometrika 49:141-9.

Rupnik M, Zorec R (1992) Cytosolic Chloride Ions Stimulate $\mathrm{Ca}^{2+}$-induced Exocytosis. FEBS Lett 303:221-3.

Tanaka S, Nomizu M, Kurosumi K (1991) Intracellular Sites of Proteolytic Processing of Pro-opiomelanocortin in Melanotrophs and Corticotrophs in Rat Pituitary. J Histochem Cytochem 39:809-21.

Tooze SA (1991) Biogenesis of Secretory Granules. Implications Arising from the Immature Secretory Granule in the Regulated Pathway of Secretion. FEBS Lett 285:220-4.

Tooze SA, Flatmark T, Tooze J, Huttner WB (1991) Characterization of the Immature Secretory Granule, an Intermediate in Granule Biogenesis. J Cell Biol 115:1491-1503.

Tooze J, Tooze SA (1986) Clathrin-coated Vesicular Transport of Secretory Proteins During the Formation of ACTH-containig secretory granules in AtT20 cells. J Cell Biol 103:839-50.

Weibel ER (1979) Stereological Methods for Miological Morphometry. New York: Academic Press, 67-185.

Zupančič G, Kocmur L, Veranič P, Grilc S, Kordaš M, Zorec R (1994) The Separation of Exocytosis from Endocytosis in Rat Melanotroph Membrane Capacitance Records. J Physiol (Lond) 480.3:539-52. 